\title{
Torsional MEMS Magnetometer Operated off-Resonance for in-Plane Magnetic Field Detection
}

\author{
G. Laghi*, S. Dellea*, A. Longoni*, P. Minotti*, A. Tocchio ${ }^{+}$, S. Zerbini ${ }^{+}$, G. \\ Langfelder* \\ * Politecnico di Milano, Dipartimento di Elettronica, Informazione e Bioingegneria, via \\ Ponzio 34/5, 20133, Milano, Italy \\ ${ }^{+}$ST Microelectronics, AMS Division, via Tolomeo 1, 20010, Cornaredo (MI), Italy
}

\begin{abstract}
The work presents a microelectromechanical system (MEMS) based magnetometer, targeting compass applications performance, which measures magnetic fields along an in-plane direction. The magnetometer is fabricated with the surface micromachining process used for consumer gyroscopes, accelerometers, and recently proposed out-of-plane magnetometers. The magnetometer is based on the Lorentz force principle, so to avoid the need for magnetic materials integration. It features an area of $282 \times 1095 \mu \mathrm{m}^{2}$, and it is wafer-wafer packaged at a nominal pressure $(0.35$ mbar $)$ similar to the one used for gyroscopes. In agreement with theoretical predictions, operation is demonstrated both at-resonance and off-resonance: in both situations the measured resolution, normalized to unit bandwidth and applied Lorentz current, is about $120 \mathrm{nT} \cdot \mathrm{mA} / \sqrt{\mathrm{Hz}}$, but the maximum sensing bandwidth is extended from $4 \mathrm{~Hz}$ (at resonance) to 42 $\mathrm{Hz}$ in off-resonance mode, which copes with consumer specifications. Within magnetic fields of $\pm 5 \mathrm{mT}$, the device shows measured linearity errors $<0.5 \%$ of the full-scale-range (demonstrating a large linearity) and a cross-axis rejection of $\sim 50 \mathrm{~dB}$. The bias stability in off-resonance operation mode $(80 \mathrm{nT} \cdot \mathrm{mA}$ at $100 \mathrm{~s}$ ) improves by a factor 100 with respect to resonance operation.
\end{abstract}

Keywords: MEMS, magnetometers, inertial measurement units, digital compass

Preprint submitted to Sensors and Actuators A

December 9, 2014 
2014 MSC: 00-01, 99-00

\section{Introduction}

The increasing interest towards multi-axis, multi-parameter measurement units [1, where different devices are integrated on the same die, drives the research on miniaturized magnetometers compatible with industrial MEMS processes. Magnetometers can be used to determine the absolute orientation, so for heading purposes, and to improve navigation capabilities when coupled to gyroscopes and accelerometers. Though some works propose the integration of magnetic thin layers on top of suspended MEMS structures [2, most of the literature about MEMS magnetometers rely on the Lorentz force principle, as this concept allows avoiding magnetic materials and the corresponding need for relatively low temperature during the fabrication steps (some magnetic materials begin losing their properties above about $300{ }^{\circ} \mathrm{C}$ ). Early works [3] and several thereafter [4, 5, 6] mostly focused on Lorentz-force devices to measure out-of-plane magnetic fields. Few examples of in-plane (either differential or non-differential) MEMS magnetometers were also described in the literature [7, 8, 9, 10, 11, 12, 13. The performances of all these devices, from a system point of view, are limited by a critical trade-off between resolution and bandwidth when operated at resonance: the former improves, the latter worsens with decreasing damping coefficients (obtainable e.g. by lowering the package pressure down to values compatible with high-quality-factor gyroscope operation). Among the strategies to overcome this limitation, a way to improve the resolution was proposed in [4]: the use of multiple loops for current re-circulation directly increases the sensitivity (and in turn the resolution) by the number of added loops. This technique is however not directly compatible with industrial surface micromachining, as it requires the deposition of metals on suspended springs. A second proposed strategy is the use of parametric amplification [14, where the device stiffness is modulated at twice the device resonance frequency. The intrinsic need for added circuitry to generate and control this added sine 
wave implies added power consumption, and it is therefore not in the direction

of low-power solutions. Another solution to extend the bandwidth is the use of frequency modulated (FM) operation [15] . Recently, off-resonance operation was also proposed [16] as an effective alternative to solve the mentioned trade-off, still using an amplitude modulated (AM) approach.

This off-resonance technique is here applied to a novel single-axis, differential, torsional magnetometer, sensitive to an in-plane direction of the magnetic field. The device preliminary characterization was presented in [17. Capacitive sensing can be performed through differential parallel plates designed beneath the suspended, tilting mass. The device description is given in details in Section II. The structure geometry is designed in order to have a pronounced form

40 factor, (i) to exploit long current paths, which directly determine the Lorentz force, and (ii) to cope with the possible integration of two of such devices in a single die together with 3 -axis gyroscopes, 3 -axis accelerometers, and the Z-axis magnetometer [16]. Fig. 1 is a possible arrangement of such devices in a 9-axis inertial measurement unit (IMU), which is derived from a typical floorplan of a 6-axis unit: a single-mass, 3-axis gyroscope is designed (as a single drive stage saves power consumption), and the accelerometers are placed in a separate cavity to operate at a different pressure. The overall IMU area taken up by this solution may be smaller than in situations where a single 3-axis magnetometer is designed (see e.g. the implementation in [7]).

The device of this work is coupled to a custom readout electronic board and tested both at resonance and with driving frequencies slightly below resonance (according to [16]), under AC and DC varying magnetic fields. As expected, the overall analog sensitivity (output voltage per unit magnetic field change) decreases linearly with the frequency mismatch, according to the theory discussed in Section III. With proper electronic filtering however (see Section IV), thermomechanical noise also decreases by the same amount, so that the resolution per unit bandwidth and unit current consumption (as defined in [18]) does not worsen $(\approx 120 \mathrm{nT} \cdot \mathrm{mA} / \sqrt{\mathrm{Hz}}$ is measured in both cases $)$, as experimentally demonstrated in Section V. Long-term acquisitions (10 minutes) reveal a much 
larger stability in off-resonance operation mode (about $1.6 \mu \mathrm{T}$ at $50 \mu \mathrm{A}$ drive current) than for resonant operation, thanks to the insensitivity to bias fluctuations.. Furthermore, the measured $\pm 3 \mathrm{~dB}$ bandwidth under AC magnetic fields, increases by a factor 10, from the quality-factor-limited value of $4 \mathrm{~Hz}$ at resonance to more than $40 \mathrm{~Hz}$ in off-resonance mode. Such a bandwidth value, together with the good linearity and cross-axis rejection measurements, make the device compatible with consumer-grade specifications.

\section{Devices description and finite element model (FEM) simulation}

A sketch of the device working principle with a detail captured at the scanning electron microscope (SEM) is shown in Fig. 2.

\subsection{Process and mechanical design}

The device is fabricated with the ThELMA (thick epitaxial layer for microactuators and accelerometers) technology from ST-Microelectronics, which consists of an epitaxial growth of a $22-\mu$ m-thick layer of heavily doped polycrystalline silicon that, once etching and release are performed, forms the suspended frame of the device. Suspension is obtained through suitable torsional springs. Beneath the suspended structure, and electrically isolated from the substrate through an oxide layer, a thin polysilicon layer is used as a bottom electrode for differential parallel-plate capacitive sensing $\left(C_{1}, C_{2}\right)$. In order to minimize the Brownian noise, the device is packaged at a relatively low pressure (nominally 0.35 mbar), through the use of getter materials and glassfrit bonding. The reader can find more details on the process e.g. in [19].

In details, the structure consists of four torsional springs sustaining a $1095 \mu \mathrm{m}$. $282 \mu \mathrm{m}$ suspended plate, as represented in Fig. 2 b. The springs are all designed along the rotational axis of the device, and their optimization considers both 85 the mechanical and electrical aspects involved in the device working principle: on one side, they are designed with a relatively short length (51 $\mu \mathrm{m}$ each fold), to shift the in-plane translational mode to high frequencies; their layout width 
$(3.7 \mu \mathrm{m})$ is chosen in such a way that the total electrical resistance (seen by the current generator) is in the order of $1.4 \mathrm{k} \Omega$. Two springs are indeed used as current inlets, and two are used as current outlets. In this way, by injecting a current through the springs, which then flows along opposite x-directions, in presence of an external magnetic field along the y-axis, a Lorentz forces pair arises, which determines a torque on the suspended plate along its longitudinal axis.

Suitable cuts in the geometry direct the injected current along the most lateral part of the frame, so to maximize the generated torque. If the lumped coefficient $\alpha$ is defined to take into account the effectiveness of the applied force $(\alpha=1$ for a force applied at the plate lateral ends, $\alpha=0.5$ for a force evenly distributed on the plate area), from finite element (FE) electrical simulations such cuts allow to have an $\alpha$ coefficient in the order of 0.8 .

The device is designed in order to have the first mechanical mode (Fig. 3) at a nominal frequency of $20 \mathrm{kHz}$, just out of the acoustic bandwidth and above the typical vibration bandwidth, to avoid related disturbances (a very common approach also in other inertial sensors [20]). The motion is detected by the parallel-plate capacitors (Fig. 2k), with an area of $7.9 \cdot 10^{4} \mu \mathrm{m}^{2}$, suitably designed $2 \mu \mathrm{m}$ below the frame (the corresponding buried electrical interconnections can be seen in the SEM detail of Fig. 2 a).

The first mode corresponds to a differential capacitance variation that is readout by a suitable electronics. Parametric FE simulations were also performed to check the stability of the frequency against process nonuniformities. The relatively wide springs make the device well immune to typical over- or underetching values. Apart from etching nonuniformities, the process height may vary as a result of a non perfect planarization. The results of the FE simulations indicate a relative variation in the first mode frequency of $\pm 0.7 \%$ only, for a maximum relative thickness variation of $\pm 4.6 \%( \pm 1 \mu \mathrm{m}$ around a nominal height of $22 \mu \mathrm{m})$.

It is also important that high-order modes occur at frequencies much larger than the mode of interest, to help rejecting possible effects of in-plane or out-of-plane 
accelerations. In the proposed design, the structure second mechanical mode is an in-plane translation which occurs at high frequency (about $78 \mathrm{kHz}$ ) thanks to the width of the designed springs, and ideally produces no variations in both the capacitors. The third mode (at about $92 \mathrm{kHz}$ ) is an out-of-plane mode that produces the same nominal capacitance variation on the two capacitors: it is therefore rejected by the electronics because it is seen as a common-mode signal. These high-order modes are shown in Fig. 4

\subsection{Capacitance variation per unit displacement}

As the sensing capacitor is here not an ideal capacitor, but rather a perforated torsional plate, $\mathrm{FE}$ electrical simulations were performed to evaluate the variation of the sensing capacitance per unit displacement of the structure. The results will be later used to predict the overall device sensitivity (output voltage variation per unit magnetic field change) and compare it to experimental data. The simulation includes the relevant parameters for the capacitance calculation, like the nominal distance between the suspended plate and the readout electrodes $(1.8 \mu \mathrm{m})$, the thickness of the oxide between the readout electrodes and the substrate $(2.6 \mu \mathrm{m})$, and the distance between the package and the top face of the suspended mass $(5 \mu \mathrm{m})$. The dielectric constant of the gas inside the package volume is reasonably assumed as that of vacuum.

The results are reported in Fig 5 a, showing the absolute value of the two singleended capacitances as a function of the maximum vertical displacement (i.e. of one lateral end of the plate). A deviation with respect to the best linear fitting is visible. However in differential mode (Fig 5 5),$C_{1}-C 2$, the linearity error is lower than $0.4 \%$ for displacements as large as $150 \mathrm{~nm}$, with a fully differential sensitivity $\frac{d C}{d Z}=0.225 \mathrm{fF} / \mathrm{nm}$. Note that such displacement values are lower than the maximum displacement to which the structure is subject in the operating conditions described in the following of this work.

Table 1 summarizes the design parameters of the torsional magnetometer [inserire tabella]. 


\section{Theory for off-resonance operation}

Off-resonance or mode-split operation is defined as the operation of a sensor

150

known technique to extend the sensing bandwidth in gyroscopes, by designing on purpose the drive mode at a frequency below the sense mode 21]. It was first proposed for magnetometers in [18, using a frequency of the Lorentz current below the resonance frequency. The split between driving and resonance an external magnetic field $B_{\text {ext }}$ along the shown direction, a pair of Lorentz forces arises, each with a modulus

$$
\begin{gathered}
F_{L}(t)=i(t) \cdot B_{\text {ext }} \cdot L \\
\frac{d F_{L}(t)}{d B_{\text {ext }}}=i(t) \cdot L
\end{gathered}
$$

where $L$ is the effective length of the current path. The current flows distributed in the outer part of the frame: assuming therefore the coefficient $\alpha$ described in Section 2, and regardless of the operation mode of the device (either at or off-resonance), the displacement $z(t)$ experimented by each of the plate lateral ends can be expressed as:

$$
\begin{gathered}
z(t)=\frac{Q_{e f f}}{k} \cdot \alpha \cdot i(t) \cdot B_{e x t} \cdot L \\
\frac{d z(t)}{d F_{L}(t)}=\frac{Q_{e f f}}{k} \alpha
\end{gathered}
$$

170

where $k$ is the device stiffness (a small displacement approximation is used, so that $k=33 \mathrm{~N} / \mathrm{m}$ is here intended as the ratio between a DC or quasi-stationary 
force applied at each plate lateral end, and its corresponding displacement).

$Q_{\text {eff }}$ represents the displacement amplification with respect to DC operation, and is a direct consequence of the equation describing the modulus of the maximum vertical displacement as a function of the force in the Fourier domain $(\omega$ being the angular frequency and $\omega_{0}=2 \cdot \pi \cdot f_{0}$ ):

$$
\left|\frac{z(j \omega)}{F_{L}(j \omega)}\right|=\frac{1}{m} \frac{1}{\sqrt{\omega_{0}^{4}+\omega^{4}-2 \omega_{0}^{2} \omega^{2}+\left(\frac{\omega_{0} \omega}{Q}\right)^{2}}}
$$

For the device operated at the angular resonance frequency $\omega_{0}, Q_{e f f}$ exactly corresponds to the quality factor $Q$, which depends on the damping coefficient $b$ at the typical operating pressures. For the device operated off-resonance (e.g. by a split $\Delta f$ in the order of $50-100 \mathrm{~Hz}$ below a resonance of $20 \mathrm{kHz}), Q_{\text {eff }}$ represents an effective amplification factor. $Q_{e f f}$, within a suitable range of mismatches $\Delta f$, is independent on the damping coefficient and only depends on $f_{0}$ and $\Delta f[18$ :

$$
Q_{e f f}=\frac{\omega_{0}}{2 \Delta \omega}=\frac{f_{0}}{2 \Delta f}
$$

For more details on off-resonance operation, the reader can refer to [18].

Assuming that the system is limited by the Brownian noise of the device, the expression of the intrinsic signal-to-noise ratio (SNR) and of the minimum measurable magnetic field per unit bandwidth and current consumption $\sigma_{B}$ (e.g. in units of $\mathrm{nT} \cdot \mathrm{mA} / \sqrt{\mathrm{Hz}}$ ) turns out to be identical for both the operation modes [16]:

$$
S N R=\frac{i \cdot B \cdot L}{\sqrt{4 \cdot k_{b} \cdot T \cdot b \cdot B W}} \quad \sigma_{B}=\frac{B_{\min } \cdot i_{0}}{\sqrt{B W}}=\frac{2}{L} \sqrt{k_{b} \cdot T \cdot b}
$$

For the expressions above, the remarkable difference between resonance and off-resonance operation is in the meaning of the system bandwidth $B W$ : for resonance operation, $B W$ is limited by the narrow $-3 \mathrm{~dB}$ mechanical bandwidth of the device $B W_{\text {mech }}=f_{0} /(2 Q)=b /(4 \pi m)$; for off-resonance operation, $B W$ represents an electronic filtering at a frequency typically chosen in the order of $\Delta f$. Therefore, for resonance operation, a trade-off between the minimum measurable field $\sigma_{B}$ and the system bandwidth $B W$ arises: it is e.g. possible 
to improve $\sigma_{B}$ decreasing the damping coefficient $b$, yet this determines an unavoidable reduction of the bandwidth. As both required noise and bandwidth are usually determined by the application (e.g. a few hundred nT and simultaneously at least a few tens $\mathrm{Hz}$ in the consumer field), the conclusion is that it is difficult to match the target specifications using AM resonance-operated Lorentz force magnetoemters.

On the contrary, off-resonance operation allows the bandwidth to be independent on the damping coefficient: it is therefore possible to improve the resolution by decreasing $b$, without affecting the system bandwidth; or it is possible to keep the same resolution (the same damping coefficient) with an extended bandwidth.

As anticipated above, this discussion is valid if the system is limited by the MEMS thermomechanical noise. If we include the electronic noise $\sigma_{\text {eln }}^{2}$ (in $\left.\left[V^{2} / H z\right]\right)$, Eq. (7) becomes:

$$
\sigma_{B}=\frac{2}{L} \sqrt{k_{b} \cdot T \cdot b+\sigma_{\text {eln }}^{2} \frac{k^{2}}{4 \cdot Q_{\text {eff }}^{2} \cdot \frac{d C}{d z} \frac{d V}{d C}}}
$$

where the electronic gain $\frac{d V}{d C}$ is introduced in the next section. The equation clearly shows that a large $Q_{e f f}$ is advantageous to make the electronic noise negligible. Off-resonance operation therefore requires a lower noise readout electronics than resonant operation to effectively hold the same SNR.

\section{Measurement setup}

With the purpose of obtaining a low-noise readout, the front-end of this work is implemented using the circuit presented in Fig. 6, based on board-level charge amplifiers (Fig. 6), a lock-in amplifier that provides the reference current and performs the demodulation and a LabVIEW acquisition interface [16] (in the future perspective of a miniaturized system, the lock-in reference signal can be substituted by a signal generated by a MEMS resonator, and the demodulation can be performed with custom circuits). 
As explained in Section II, if the device is subject to a magnetic field that has a component in the sensing direction, a Lorentz-force pair arises, causing a differential capacitance variation between the tilting mass and the sensing plates on the substrate. The two sensing plates are held at a fixed potential $(6 \mathrm{~V})$ thanks to the virtual ground of the two charge amplifier stages to which they are directly connected. These charge amplifiers, implemented through AD8065 operational amplifiers, $C_{F}=0.6 \mathrm{pF}$ feedback capacitances and $R_{F}=600 \mathrm{M} \Omega$ feedback resistances, are used to convert the current flowing out of the MEMS stators to voltage signals at their outputs. These signals (nominally with the same amplitude and a relative phase shift of $\pi$ ) are further amplified through an instrumentation amplifier (INA 129), in order to reject any common mode component. At this output there is a sinusoidal signal, whose amplitude is proportional to the external magnetic field. In order to obtain a DC voltage at the end of the readout chain, a demodulation is performed with a lock-in amplifier, with a low-pass bandwidth that can be selected according to the needs of the specific measurement. Regarding the electronic noise of the whole readout chain, it turns out to be limited by the thermal noise of the feedback resistances $\left(R_{F}\right)$ of the charge amplifier stages.

For the generation of the driving current through the magnetometer, a balanced, differential Howland current pump circuit is used, as shown in Fig. 6 . which converts the sinusoidal voltage coming from the lock-in amplifier into a 245 sinusoidal current signal, which is injected into the sensor.

Apart from the useful signal, an offset contribution arises at the output, likely due to simultaneous resistivity unbalance of the current paths, and a mechanical offset at rest for the suspended mass. The offset origin is currently under deeper investigation. Though the driving circuit is set in order to minimize this offset, 250 a residual contribution in the order of $1 \mathrm{mT}$ is observed (both at resonance and off-resonance) and compensated through a post-acquisition subtraction (hardware offset compensation techniques were discussed in 22]). The stability of this offset will be analyzed in the next subsection.

The board with the magnetometer is mounted within a three-axial Helmholtz- 
terfaces, to generate arbitrary-shape fields, with a maximum frequency of $\sim 200$

The described electronics gives an overall capacitive sensitive gain described by the equation below:

$$
\frac{d V_{\text {out }}(t)}{d C(t)}=\frac{V_{\text {bias }}}{C_{F}} * G_{I N A} * G_{L I A}=2.9 \mathrm{~V} / f F
$$

260 the product of the Instrumentation Amplifier and Lock-in Amplifier gains. It is now possible to put together equations (2), (4), (8) and the results of FE simulations of Section $2 \mathrm{~b}$ to derive the theoretical expression of the sensitivity in both operation modes:

$$
S=\frac{d V_{\text {out }}}{d C} \cdot \frac{d C}{d Z} \cdot \frac{d z}{d F_{L}} \cdot \frac{d F_{L}}{d B_{\text {ext }}}
$$

265

\section{Experimental results}

\subsection{Sensitivity and linearity}

In order to characterize the device and to validate the proposed theory, a sensitivity analysis is first performed by injecting the current at the measured resonance frequency $f_{0}=19953 \mathrm{~Hz}$, and generating a quasi-static ramp of magnetic fields from $-5 \mathrm{mT}$ to $+5 \mathrm{mT}$, consecutively along the three Cartesian axes. The measured output voltage values at the end of the acquisition chain are shown in Fig. 7. A sensitivity of $0.85 \mathrm{~V} / \mathrm{mT}$ is obtained for the sensitive axis, which is compatible with a full-scale range of a few $\mathrm{mT}$ at reasonably low voltages. This value matches the predictions made at the end of Section IV, 
is obtained, which is again in agreement with the predictions of Section 4 using the same coefficient $\alpha$ considered for resonant operation.

\subsection{Noise and long-term stability}

305

A noise analysis is then performed, by holding the device inside a zero-gausschamber and by varying the demodulation frequency from around resonance to $\sim 500 \mathrm{~Hz}$ of mismatch, with a very narrow lock-in low-pass-filter (LPF) bandwidth. Fig. 93 shows the measured results, where one can note that at frequency mismatches higher than $\approx 100 \mathrm{~Hz}$ the noise becomes significantly limited by the 

for resonance operation, (i) the effect of the narrow bandwidth is clearly visible at small observation times, and (ii) huge drifts occur after a few s of observation. 
The development of a suitable driving and readout ASIC to implement an autonomous magnetic field sensing system represents ongoing work. To be competitive with other technologies, an overall power consumption of less than 0.5 
$\mathrm{mW}$ per axis is required. Assuming a voltage in the order of $1.7 \mathrm{~V}$, the overall

current consumption of $294 \mu A_{r m s}$ can be in a first approximation equally split between the device and the ASIC. This results in $210 \mu \mathrm{A}$ peak current available for the design of a low-noise ASIC.

\section{Acknowledgments}

The author thank N. Aresi for helping with the initial device characterization. This work was supported by the European Union under the ENIAC Grant n. 325622 (Lab4MEMS project).

\section{References}

[1] Yole Developpement, Technologies and sensors for the Internet of Things: Businesses \& Market Trends 2014-2024, Publication Jun. 18, 2014. Available online at www.i-micronews.com.

[2] D. Ettelt, P. Rey, G. Jourdan, A. Walther, P. Robert, J. Delamare, 3D Magnetic Field Sensor Concept for Use in Inertial Measurement Units (IMUs), Microelectromechanical Systems, Journal of 23 (2) (2014) 324-333.

[3] H. Emmerich, M. Schofthaler, U. Knauss, A novel micromachined magnetic-field sensor, in: Micro Electro Mechanical Systems, 1999. MEMS '99. Twelfth IEEE International Conference on, 1999, pp. 94-99.

[4] J. Kyynarainen, J. Saarilahti, H. Kattelus, A. Karkkainen, T. Meinander, A. Oja, P. Pekko, H. Seppa, M. Suhonen, H. Kuisma, S. Ruotsalainen, M. Tilli, A 3D micromechanical compass, Sensors and Actuators A: Physical 142 (2) (2008) 561 - 568, the sixth European Magnetic Sensor and Actuator conference.

[5] M. Thompson, D. Horsley, Resonant MEMS magnetometer with capacitive read-out, in: Sensors, 2009 IEEE, 2009, pp. 992-995. 
[6] G. Langfelder, C. Buffa, A. Frangi, A. Tocchio, E. Lasalandra, A. Longoni,

[12] R. Sunier, T. Vancura, Y. Li, K.-U. Kirstein, H. Baltes, O. Brand, Resonant magnetic field sensor with frequency output, Microelectromechanical Systems, Journal of 15 (5) (2006) 1098-1107.

[13] M. Li, V. Rouf, G. Jaramillo, D. Horsley, Mems lorentz force magnetic 420 Z-Axis Magnetometers for MEMS Inertial Measurement Units Using an Industrial Process, Industrial Electronics, IEEE Transactions on 60 (9) (2013) 3983-3990.

[7] M. Li, E. Ng, V. Hong, C. Ahn, Y. Yang, T. Kenny, D. Horsley, Singlestructure 3-axis lorentz force magnetometer with sub-30 nT/ $\sqrt{H Z}$ resolution, in: Micro Electro Mechanical Systems (MEMS), 2014 IEEE 27th International Conference on, 2014, pp. 80-83.

[8] C.-I. Chang, M.-H. Tsai, C.-M. Sun, W. Fang, Development of cmos-mems in-plane magnetic coils for application as a three-axis resonant magnetic sensor, Journal of Micromechanics and Microengineering 24 (3) (2014) 035016.

[9] B. Eyre, K. Pister, W. Kaiser, Resonant mechanical magnetic sensor in standard cmos, Electron Device Letters, IEEE 19 (12) (1998) 496-498.

[10] A. Herrera-May, P. Garca-Ramrez, L. Aguilera-Corts, E. Figueras, J. Martinez-Castillo, E. Manjarrez, A. Sauceda, L. Garca-Gonzlez, R. Jurez-Aguirre, Mechanical design and characterization of a resonant magnetic field microsensor with linear response and high resolution, Sensors and Actuators A: Physical 165 (2) (2011) 399 - 409.

[11] Z. Kdr, A. Bossche, P. Sarro, J. Mollinger, Magnetic-field measurements using an integrated resonant magnetic-field sensor, Sensors and Actuators A: Physical 70 (3) (1998) $225-232$.

sensor based on a balanced torsional resonator, in: Solid-State Sensors, Actuators and Microsystems (TRANSDUCERS EUROSENSORS XXVII), 
2013 Transducers Eurosensors XXVII: The 17th International Conference on, 2013, pp. 66-69.

[14] M. Thompson, D. Horsley, Parametrically Amplified Z -Axis Lorentz Force Magnetometer, Microelectromechanical Systems, Journal of 20 (3) (2011) $702-710$.

[15] M. Li, S. Sonmezoglu, D. Horsley, Extended bandwidth lorentz force magnetometer based on quadrature frequency modulation, Microelectromechanical Systems, Journal of PP (99) (2014) 1-1.

[16] G. Langfelder, G. Laghi, P. Minotti, A. Tocchio, A. Longoni, Off-Resonance Low-Pressure Operation of Lorentz Force MEMS Magnetometers, Industrial Electronics, IEEE Transactions on 61 (12) (2014) 7124-7130.

[17] S. Dellea, G. Laghi, G. Langfelder, A. Longoni, P. Minotti, A. Tocchio, S. Zerbini, Off-resonance operation of in-plane torsional MEMS magnetometers, in: Proc. of Eurosensors 2014, Brescia, Italy, 2014.

[18] G. Langfelder, A. Tocchio, Operation of Lorentz-Force MEMS Magnetometers With a Frequency Offset Between Driving Current and Mechanical Resonance, Magnetics, IEEE Transactions on 50 (1) (2014) 1-6.

[19] G. Langfelder, S. Dellea, F. Zaraga, D. Cucchi, M. Urquia, The Dependence of Fatigue in Microelectromechanical Systems on the Environment and the Industrial Packaging, Industrial Electronics, IEEE Transactions on 59 (12) (2012) 4938-4948.

[20] R. Dean, S. Castro, G. Flowers, G. Roth, A. Ahmed, A. Hodel, B. Grantham, D. Bittle, J. Brunsch, A Characterization of the Performance of a MEMS Gyroscope in Acoustically Harsh Environments, Industrial Electronics, IEEE Transactions on 58 (7) (2011) 2591-2596.

[21] S. Alper, T. Akin, A Single-Crystal Silicon Symmetrical and Decoupled MEMS Gyroscope on an Insulating Substrate, Microelectromechanical Systems, Journal of 14 (4) (2005) 707-717. 
[22] M. Li, D. Horsley, Offset suppression in a micromachined lorentz force magnetic sensor by current chopping, Microelectromechanical Systems, Journal of 23 (6) (2014) 1477-1484. 
Table 1: Device parameters

\begin{tabular}{|c|c|}
\hline Parameter & Value \\
\hline \hline Vertical gap at rest $\left(z_{0}\right)$ & $1.8 \mu \mathrm{m}$ \\
\hline Thickness $(h)$ & $22 \mu \mathrm{m}$ \\
\hline Linear stiffness $(k)$ & $33 \mathrm{~N} / \mathrm{m}$ \\
\hline Effective mass $(m)$ & $2.1 \mathrm{nkg}$ \\
\hline Resonance frequency $\left(f_{0}\right)$ & $19.95 \mathrm{kHz}$ \\
\hline Nominal package pressure $(p)$ & $0.35 \mathrm{mbar}$ \\
\hline Quality factor $(Q)$ & 2500 \\
\hline Electrical resistance $(R)$ & $1.405 \mathrm{k} \Omega$ \\
\hline \hline
\end{tabular}

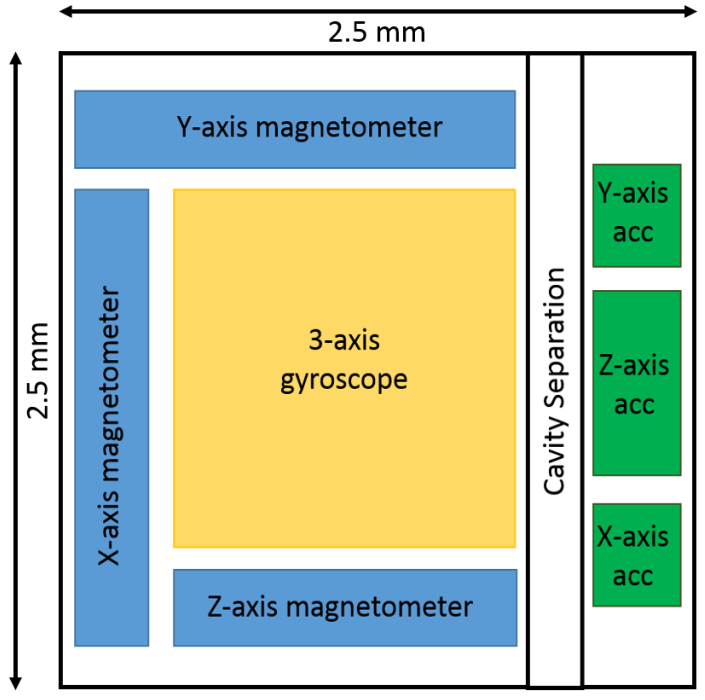

Figure 1: Schematic concept of an inertial measurement unit (IMU) integrating three singleaxis accelerometers, a 3-axis gyroscope and three single-axis magnetometers. The implementation of the gyroscope as a highly symmetric monolithic 3-axis device is guided by power consumption and quadrature error issues. The magnetometers well fit on the gyroscope sides. 

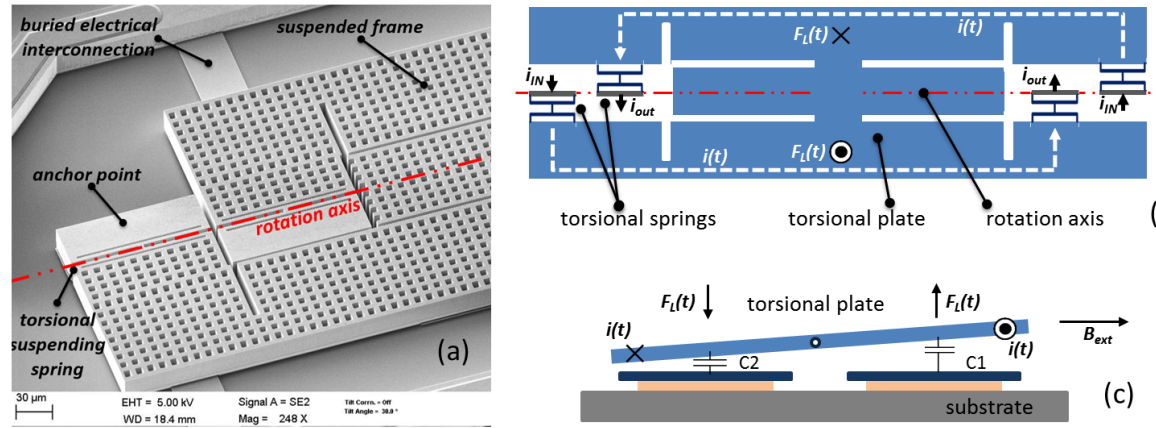

Figure 2: (a) scanning electron microscope (SEM) picture of the device proposed in this work, showing details of the torsional springs and the rotation axis. The operating principle is schematized by the top view (b) and the cross-section (c).

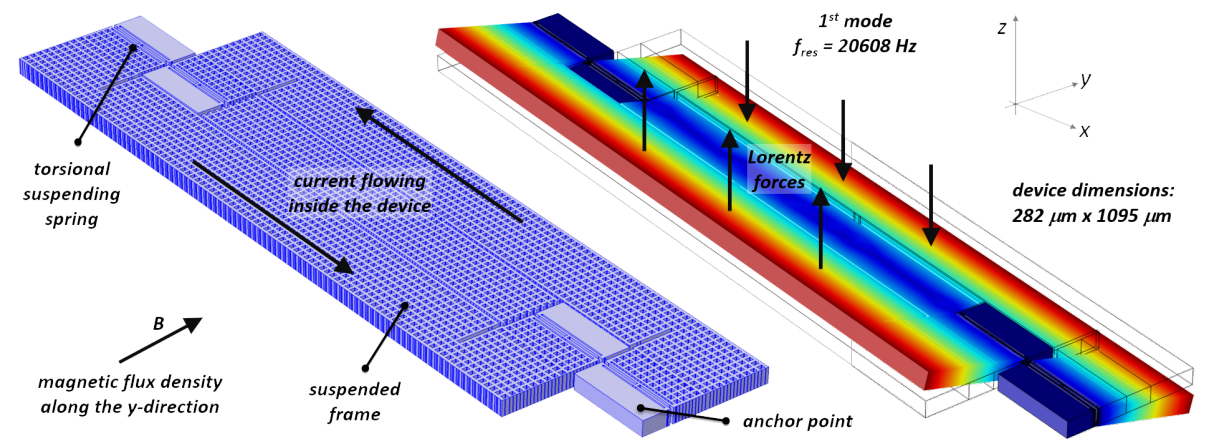

Figure 3: Device geometry and first resonant mode: the current, flowing as indicated by the arrows, causes a Lorentz torque in event of magnetic fields along the y-axis. The corresponding displacement is read by differential parallel plates designed beneath the seismic mass (not shown in the figure). 


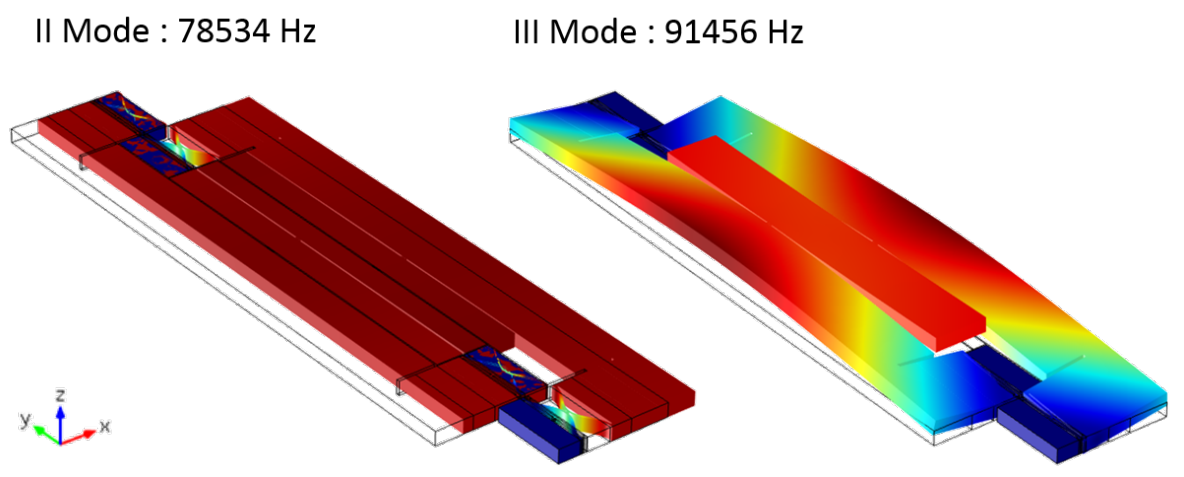

Figure 4: Second and third mechanical modes of the structure: ideally they produce no effect and a common mode effect (respectively) on the differential capacitance variation.
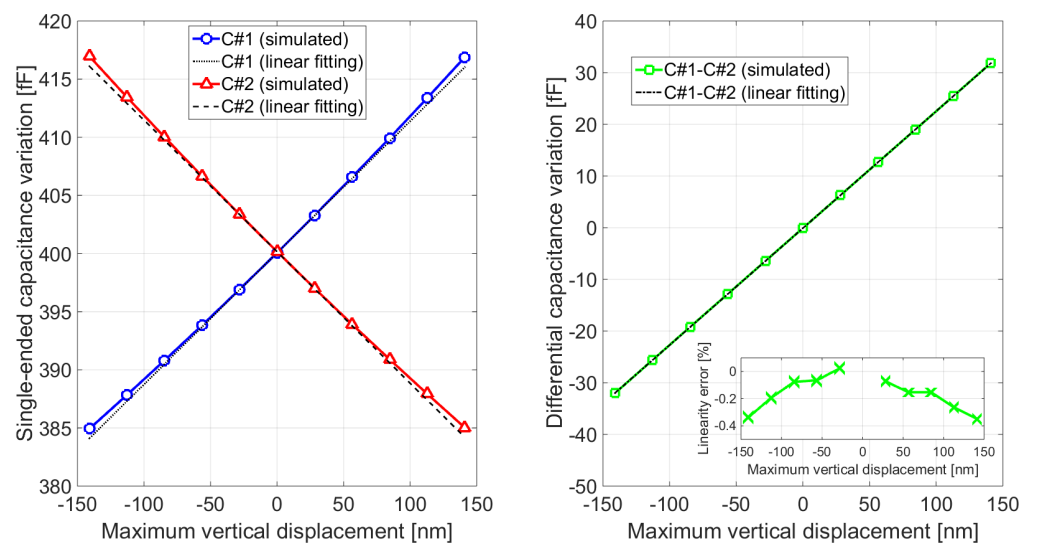

Figure 5: (a) results of FE simulations for the single-ended capacitance variation of the torsional magnetometer, and (b) results in terms of differential capacitance change (b). The inset shows the linearity error in the latter condition. 


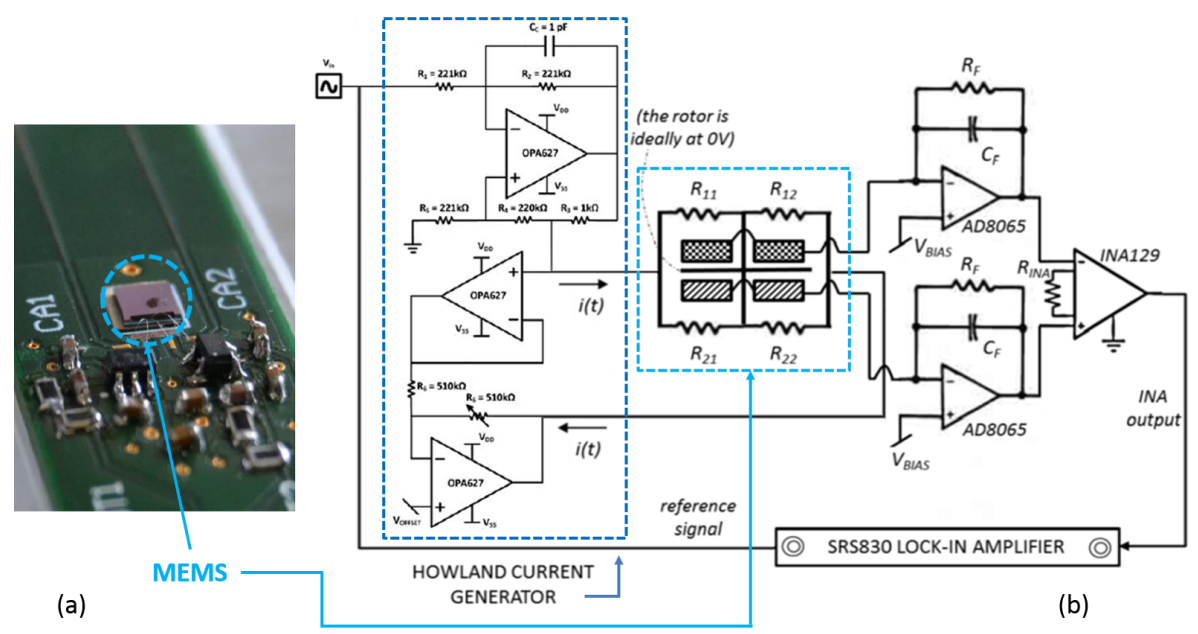

Figure 6: (a) Optical picture showing the MEMS package glued onto the printed circuit board, and the front-end of the readout electronics with the charge amplifiers; (b) complete schematic of the circuit used for the readout, where the Howland current generator, the charge amplifiers, the INA stage and the Lock-In amplifier can be identified.
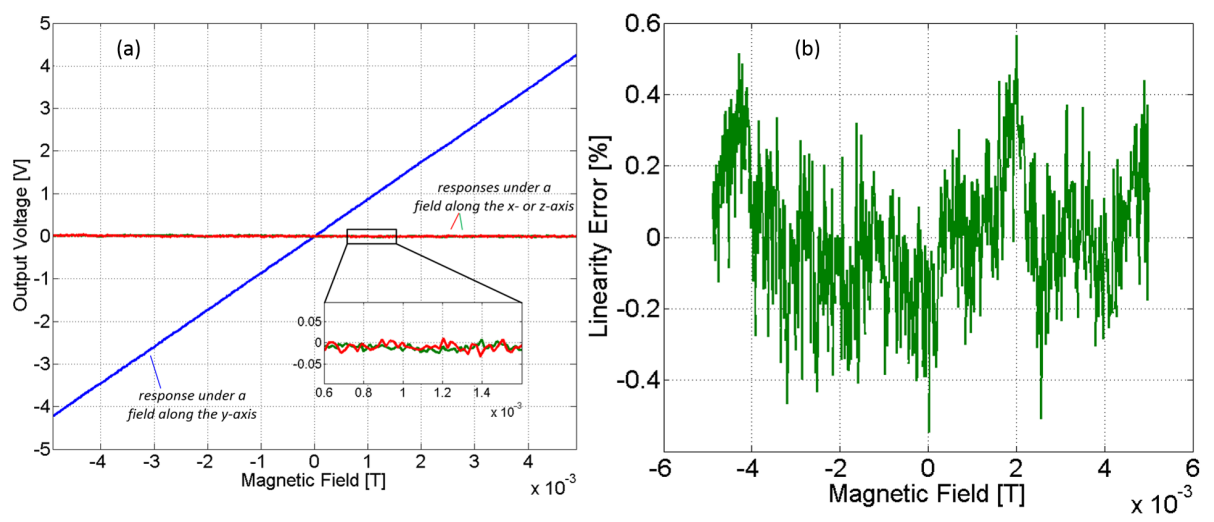

Figure 7: (a) Output voltage as a function of the magnetic field, applied subsequently along the three axes while the device is driven with a current at the resonance frequency; (b) linearity error between measured sensitivity and its best linear fitting demonstrates the very good linearity of the device. 

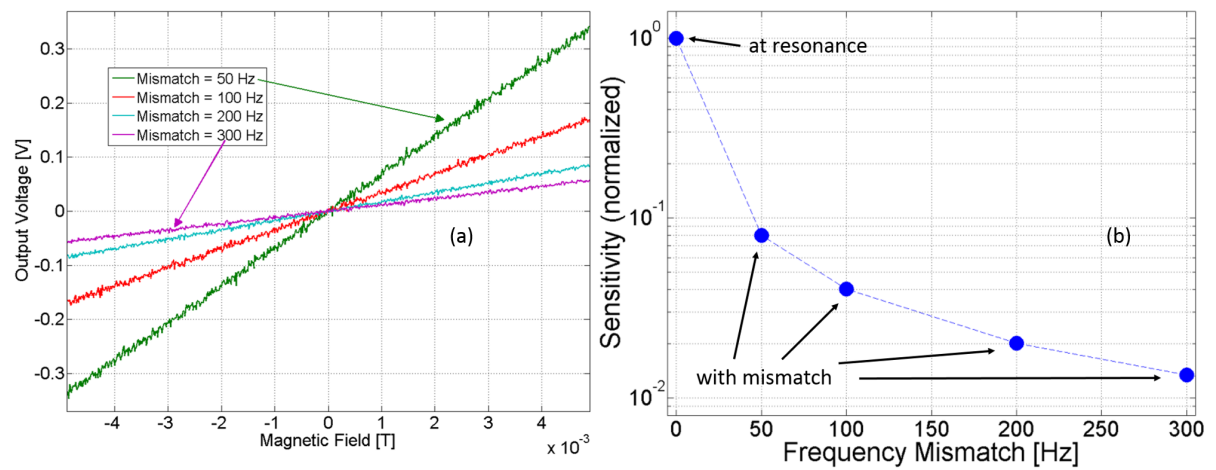

Figure 8: (a) Output voltage as a function of the magnetic field, for a current injected offresonance for different frequency mismatches; (b) the resulting sensitivity goes linearly with the inverse of the mismatch, as predicted by the theory developed in Section III.
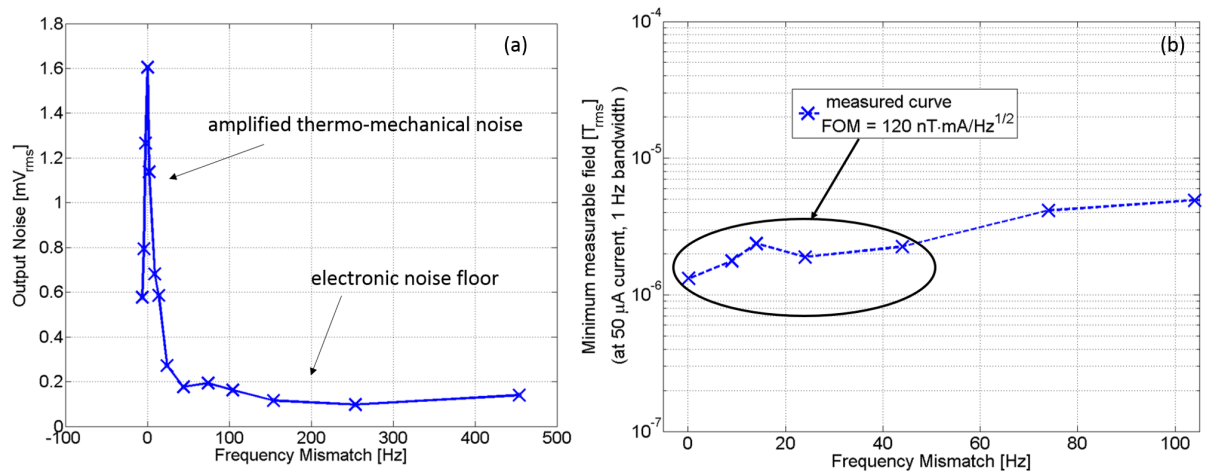

Figure 9: (a) Measured output noise as a function of the frequency mismatch. The thermomechanical contribution, amplified by the quality factor, is clearly visible at resonance. For large mismatches the noise floor is due to the electronics; (b) minimum measurable field per unit bandwidth and current consumption, from resonance to off-resonance operation: the value is almost constant up to mismatches of about $50 \mathrm{~Hz}$. 

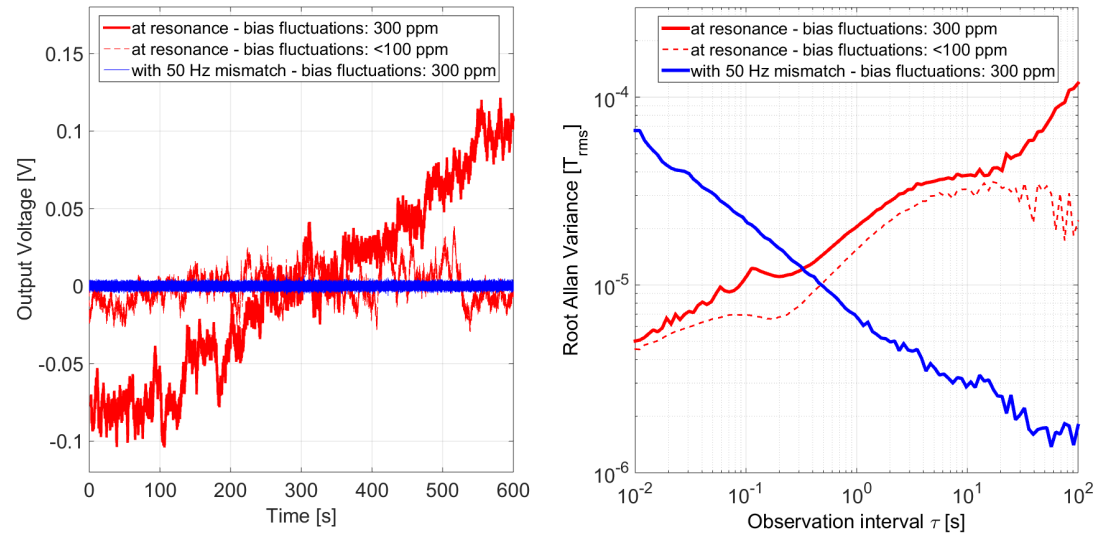

Figure 10: (a) measured output voltage vs time in different conditions of stator bias voltage stability in resonance mode, and in off-resonance operation; (b) Allan variance plots derived from the acquisition in (a).

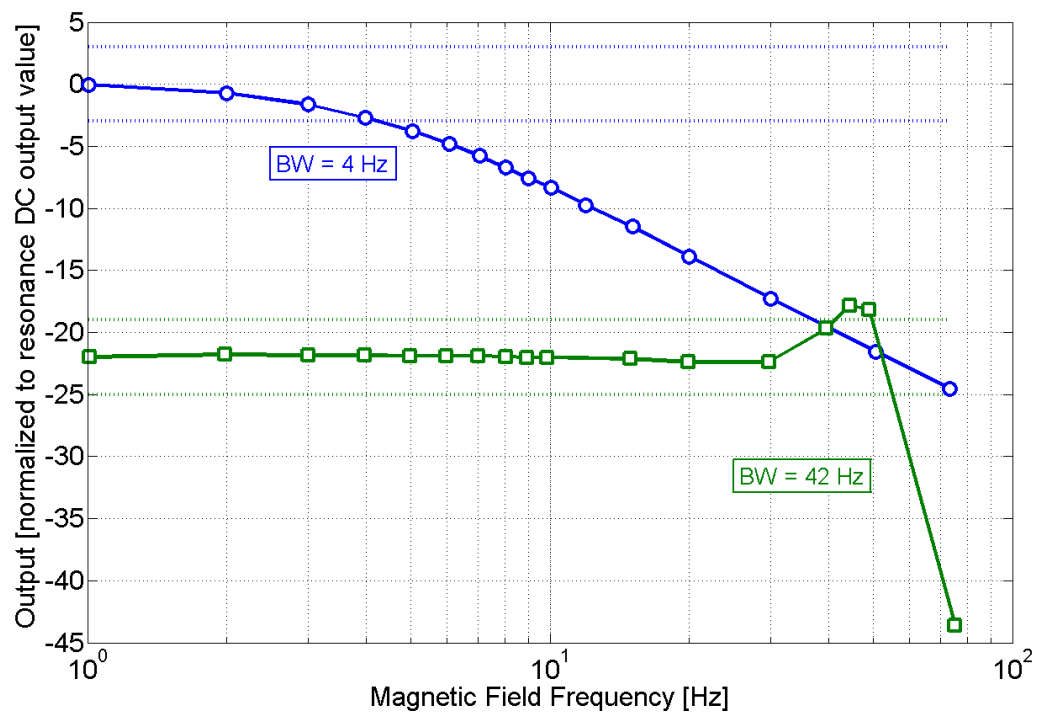

Figure 11: Sensing bandwidth measured under AC fields for resonance and off-resonance operation. The obtained bandwidth extension in off-resonance mode is larger than a factor of 10. The sensitivity-bandwidth trade-off is visible. 